\title{
La pression motrice au cours du syndrome de détresse respiratoire aiguë
}

\section{Driving Pressure in Acute Respiratory Distress Syndrome}

\author{
A. Mercat \\ Reçu le 14 novembre 2016; accepté le 24 novembre 2016 \\ (C) SRLF et Lavoisier SAS 2016
}

Fondée sur des données expérimentales solides [1] et des arguments physiopathologiques convaincants [2], la ventilation protectrice a démontré qu'elle permettait de réduire la mortalité hospitalière du syndrome de détresse respiratoire aiguë (SDRA) [3,4]. Cependant, la question du ou des critères pertinents pour l'adaptation personnalisée de cette stratégie reste débattue. C'est dans le cadre de cette problématique que Marcelo Amato a mis en exergue l'intérêt de la mesure de la pression motrice (driving pressure) qui correspond à la variation de pression alvéolaire induite par le volume courant, calculée comme : $\Delta \mathrm{P}=$ Pression de plateau - Pression Expiratoire Positive (PEP) [5]. L'hypothèse n'est pas nouvelle puisque, dès 1998, dans la publication démontrant pour la première fois le bénéfice de la ventilation protectrice en termes de survie, ce même auteur évoquait déjà le rôle de ce paramètre [6].

Les deux déterminants du $\Delta \mathrm{P}$ sont le volume courant $\left(\mathrm{V}_{\mathrm{T}}\right)$ et la compliance du système respiratoire $\left(\mathrm{C}_{\mathrm{RS}}\right): \Delta \mathrm{P}=\mathrm{V}_{\mathrm{T}} /$ $\mathrm{C}_{\mathrm{RS}}$. Or, au cours du SDRA, la compliance du système respiratoire est proportionnelle au volume pulmonaire aéré de fin d'expiration (EELV), volume disponible pour la distribution du volume courant : $\mathrm{C}_{\mathrm{RS}} \approx \mathrm{k} \times$ EELV. On peut donc considérer $\Delta \mathrm{P}$ comme un indice du strain dynamique défini comme la déformation imposée par le $\mathrm{V}_{\mathrm{T}}$ rapportée au volume initial : $\Delta \mathrm{P} \approx \mathrm{V}_{\mathrm{T}} / \mathrm{k} \times$ EELV [7].

Amato et al. ont analysé les données individuelles de 3562 patients atteints de SDRA et inclus dans des essais randomisés contrôlés portant sur la ventilation (comparaison de deux niveaux de $\mathrm{V}_{\mathrm{T}}$ ou de deux stratégies de réglage de la PEP ou les deux) [5]. L'objectif de l'étude était de rechercher la ou les associations éventuelles entre diverses variables mesurées à $\mathrm{J} 1$ et la mortalité. Le $\Delta \mathrm{P}$ était, dans cette étude, la variable ventilatoire la plus fortement associée à la mortalité. L'analyse de la mortalité hospitalière en fonction de la valeur de $\Delta \mathrm{P}$ suggérait l'existence d'un seuil situé à

\section{A. Mercat $(\square)$}

Département de réanimation médicale et médecine hyperbare,

CHU d'Angers, 4 rue Larrey, F-49933 Angers cedex 9

e-mail : alain.mercat@univ-angers.fr
$14 \mathrm{cmH}_{2} \mathrm{O}$ au-delà duquel la mortalité augmentait linéairement avec le $\Delta \mathrm{P}$. De plus, l'analyse de médiation montrait que la réduction de $\Delta \mathrm{P}$ était le principal médiateur de la réduction de mortalité associée aux modifications de réglage $\mathrm{du} \mathrm{V}_{\mathrm{T}}$ ou de la PEP résultant de la randomisation dans ces essais. Les limites de cette étude liées à la sélection des patients d'une part et à la méthodologie utilisée d'autre part ont été très bien analysées dans une étude bibliographique de la rubrique « Réactu » disponible sur le site web de la Société de réanimation de langue française (http://www.srlf.org/ reactu). Il faut convenir ici que ces données ne constituent pas une preuve formelle du lien de cause à effet entre réduction du $\Delta \mathrm{P}$ et amélioration de la survie et encore moins du bien-fondé d'une stratégie ventilatoire visant à réduire le $\Delta \mathrm{P}$. Il n'en reste pas moins vrai qu'elles incitent clairement le clinicien à prendre en compte ce paramètre facilement accessible pour l'adaptation personnalisée des réglages ventilatoires au cours du SDRA. Les deux limites majeures à son utilisation sont la présence d'efforts inspiratoires soutenus (ventilation assistée) et l'existence d'une éventuelle altération de la compliance de la paroi thoracique. En présence d'efforts inspiratoires, la mesure de la pression de plateau peut devenir très délicate, voire impossible, empêchant le calcul de $\Delta \mathrm{P}$. Il faut par ailleurs rappeler que, dans les modes en pression comme l'aide inspiratoire par exemple, la pression inspiratoire réglée ne saurait être assimilée à la pression de plateau. Cette limite ne devrait cependant pas poser de problème, au moins à la phase initiale, chez les patients atteints de SDRA sévères qui sont volontiers placés en ventilation contrôlée, fortement sédatés voire curarisés, conditions permettant une mesure fiable de la pression de plateau. L'existence d'une anomalie de la compliance de paroi est plus problématique. En effet, il est clairement démontré que, chez un certain nombre de patients atteints de SDRA, la diminution de la compliance du système respiratoire résulte non seulement d'une perte de volume pulmonaire aéré mais également d'une altération de la mécanique de la paroi thoracique [8] dont la mise en évidence nécessite le recours à la mesure de la pression osophagienne, reflet de la pression pleurale [9]. Une telle mesure permet le calcul 
de la pression motrice transpulmonaire : $\Delta \mathrm{P}_{\mathrm{L}}=\left(\mathrm{P}_{\text {plateau }}-\right.$ $\left.\mathrm{P}_{\text {oeso inspiratoire }}\right)-\left(\mathrm{PEP}-\mathrm{P}_{\text {oeso expiratoire }}\right)$ qui constitue une mesure plus fiable du stress dynamique induit par le volume courant [10]. Il faut cependant noter qu'en cas d'élévation de la pression pleurale sans anomalie de la compliance de la paroi thoracique comme c'est le plus souvent le cas, par exemple, chez les patients souffrant d'obésité morbide, alors que la pression de plateau perd sa pertinence en termes d'évaluation de la pression de distension pulmonaire, le $\Delta \mathrm{P}$ garde toute sa validité comme indicateur du stress dynamique [10].

En pratique, il apparaît aujourd'hui raisonnable, après établissement d'une ventilation protectrice conforme aux recommandations actuelles, de mesurer le $\Delta \mathrm{P}$ et quand celui-ci est supérieur à $14 \mathrm{cmH}_{2} \mathrm{O}$, d'évaluer les effets de modifications du $\mathrm{V}_{\mathrm{T}}$ et/ou de la PEP sur ce paramètre, mais également sur les échanges gazeux et l'hémodynamique. L'impact clinique d'une adaptation des réglages du respirateur fondée sur la mesure de la pression œsophagienne pour le calcul du $\Delta \mathrm{P}_{\mathrm{L}}$ reste à démontrer.

Liens d'intérêts : L'auteur déclare ne pas avoir de lien d'intérêt.

\section{Références}

1. Dreyfuss D, Saumon G, (1998) Ventilator-induced lung injury: lessons from experimental studies. Am J Respir Crit Care Med 157: 294-323.

2. Gattinoni L, Marini JJ, Pesenti A, Quintel M, Mancebo J, Brochard L, (2016) The "baby lung" became an adult. Intensive Care Med 42: 663-73.

3. (2000) Ventilation with lower tidal volumes as compared with traditional tidal volumes for acute lung injury and the acute res- piratory distress syndrome. The Acute Respiratory Distress Syndrome Network. N Engl J Med 342: 1301-8.

4. Briel M, Meade M, Mercat A, Brower RG, Talmor D, Walter SD, Slutsky AS, Pullenayegum E, Zhou Q, Cook D, Brochard L, Richard JC, Lamontagne F, Bhatnagar N, Stewart TE, Guyatt G, (2010) Higher vs lower positive end-expiratory pressure in patients with acute lung injury and acute respiratory distress syndrome: systematic review and meta-analysis. JAMA 303: 865-73.

5. Amato MB, Meade MO, Slutsky AS, Brochard L, Costa EL, Schoenfeld DA, Stewart TE, Briel M, Talmor D, Mercat A, Richard JC, Carvalho CR, Brower RG, (2015) Driving pressure and survival in the acute respiratory distress syndrome. $\mathrm{N}$ Engl $\mathrm{J}$ Med 372: 747-55.

6. Amato MB, Barbas CS, Medeiros DM, Magaldi RB, Schettino GP, Lorenzi-Filho G, Kairalla RA, Deheinzelin D, Munoz C, Oliveira R, Takagaki TY, Carvalho CR, (1998) Effect of a protectiveventilation strategy on mortality in the acute respiratory distress syndrome. N Engl J Med 338: 347-54.

7. Protti A, Andreis DT, Monti M, Santini A, Sparacino CC, Langer T, Votta E, Gatti S, Lombardi L, Leopardi O, Masson S, Cressoni M, Gattinoni L, (2013) Lung stress and strain during mechanical ventilation: any difference between statics and dynamics? Crit Care Med 41: 1046-55.

8. Gattinoni L, Pelosi P, Suter PM, Pedoto A, Vercesi P, Lissoni A, (1998) Acute respiratory distress syndrome caused by pulmonary and extrapulmonary disease. Different syndromes? Am J Respir Crit Care Med 158: 3-11.

9. Mauri T, Yoshida T, Bellani G, Goligher EC, Carteaux G, Rittayamai N, Mojoli F, Chiumello D, Piquilloud L, Grasso S, Jubran A, Laghi F, Magder S, Pesenti A, Loring S, Gattinoni L, Talmor D, Blanch L, Amato M, Chen L, Brochard L, Mancebo J; PLeUral pressure working Group (PLUG-Acute Respiratory Failure section of the European Society of Intensive Care Medicine), (2016) Esophageal and transpulmonary pressure in the clinical setting: meaning, usefulness and perspectives. Intensive Care Med 42: 1360-73.

10. Loring SH, Topulos GP, Hubmayr RD (2016) Transpulmonary Pressure: The Importance of Precise Definitions and Limiting Assumptions. Am J Respir Crit Care Med [in press] 